\title{
Ultrasound guidance versus the blind method for intrauterine catheter insemination: A randomized controlled trial
}

\author{
Sarah Mubarak', Noor Haliza Yusoff', Tassha Hilda Adnan² \\ 'Reproductive Medicine Unit, Department of Obstetrics and Gynaecology and ${ }^{2}$ National Clinical Research Centre, General Hospital Kuala Lumpur, Kuala \\ Lumpur, Malaysia
}

Objective: The primary objective of this study was to compare clinical pregnancy rates in intrauterine insemination (IUI) treatment cycles with transabdominal ultrasound guidance during intrauterine catheter insemination (US-IUI) versus the "blind method" IUI without ultrasound guidance (BM-IUI). The secondary objective was to compare whether US-IUI had better patient tolerability and whether US-IUI made the insemination procedure easier for the clinician to perform compared to BM-IUI.

Methods: This was a randomized controlled trial done at the Reproductive Medicine Unit of General Hospital Kuala Lumpur, Malaysia. We included women aged between 25 and 40 years who underwent an IUI treatment cycle with follicle-stimulating hormone injections for controlled ovarian stimulation.

Results: A total of 130 patients were recruited for our study. The US-IUI group had 70 patients and the BM-IUI group had 60 patients. The clinical pregnancy rate was $10 \%$ in both groups $(p>0.995)$ and there were no significant difference between the groups for patient tolerability assessed by scores on a pain visual analog scale $(p=0.175)$ or level of difficulty for the clinician $(p>0.995)$. The multivariate analysis further showed no significant increase in the clinical pregnancy rate (adjusted odds ratio, $1.07 ; 95 \%$ confidence interval, $0.85-1.34 ; p=0.558$ ) in the US-IUI group compared to the BM-IUI group even after adjusting for potential covariates.

Conclusion: The conventional blind method for intrauterine catheter insemination is recommended for patients undergoing IUI treatment. The use of ultrasound during the insemination procedure increased the need for trained personnel to perform ultrasonography and increased the cost, but added no extra benefits for patients or clinicians.

Keywords: Artificial insemination; Insemination; Pregnancy rates; Ultrasound; Visual analog pain scale

\section{Introduction}

Infertility has become a significant problem worldwide. Multiple treatment options are available, including intrauterine insemination (IUI), in vitro fertilization (IVF), and intracytoplasmic sperm injection

Received: Dec 5, 2018 · Revised: Mar 4, 2019 · Accepted: Mar 4, 2019

Corresponding author: Sarah Mubarak

Reproductive Medicine Unit, Department of Obstetrics and Gynaecology, General Hospital Kuala Lumpur, Jalan Pahang, Kuala Lumpur 50586, Malaysia Tel:+60-123083713 Fax:+60-326948980 E-mail:sarahmubarak@yahoo.com

This is an Open Access article distributed under the terms of the Creative Commons Attribution Non-Commercial License (http://creativecommons.org/licenses/by-nc/4.0/) which permits unrestricted non-commercial use, distribution, and reproduction in any medium, provided the original work is properly cited.
[1]. For couples with mild male factor and unexplained infertility, IUI remains the first line of treatment at most centers because it is simple, cost-effective, and provides a reasonable success rate [2-4]. However, in 2013, the UK National Institute for Health and Care Excellence recommended that IUI should not be routinely offered for couples with unexplained infertility [5]. Nonetheless, in a large randomized controlled trial (RCT) of 201 cases published in 2018, Farquhar et al. [6] concluded that IUI with ovarian stimulation is a safe and effective treatment for women with unexplained infertility, with a cumulative live birth rate of $31 \%$ vs. $9 \%$ in the expectant group.

In general, IUI success rates range between 6\% and 21.2\% [7-9]. The majority of published studies on IUI have focused on ovarian 
stimulation and seminal fluid parameters, instead of on the technique of insemination itself [10]. For IVF procedures, in 2016, the Cochrane Database of Systematic Reviews presented an analysis of 21 RCTs comparing ultrasound guidance during embryo transfer versus the blind method, which relies on the clinician's tactile senses to judge if the catheter is in the correct position, and concluded that ultrasound guidance led to higher clinical pregnancy rates and live birth rates than the blind method of embryo transfer [11]. Therefore, we sought to determine whether the same technique using transabdominal ultrasound guidance during intrauterine catheter insemination (US-IUI), compared to the blind method IUI without ultrasound guidance (BM-IUI), improved clinical pregnancy rates in IUI treatment cycles. We also attempted to determine whether US-IUI had better patient tolerability than BM-IUI, and whether it made the procedure easier for the clinician for insemination in IUI treatment cycles.

\section{Methods}

This was an RCT conducted at the Reproductive Medicine Unit of the General Hospital Kuala Lumpur from July 1, 2016 through May 30, 2018. The Medical Research and Ethics Committee of Ministry of Health Malaysia approved this study (No. NMRR-16-478-29700 IIR).

\section{Sample size calculation}

The sample size was calculated using the PS: Power \& Sample Size Calculator ver. 3.0. We needed 250 patients in each group to detect a difference with $10 \%$ significance. However, we performed an interim analysis after 2 years of patient recruitment to evaluate this study.

\section{Methodology}

All couples underwent a routine infertility assessment for day 2 or day 3 basal serum follicle-stimulating hormone (FSH) levels and serum luteinizing hormone $(\mathrm{LH})$ levels, a hysterosalpingogram or laparoscopy tubal assessment was done to access tubal patency, and seminal fluid analysis was performed for all male partners.

We included patients who were between 25 and 40 years old, had a body mass index less than $35 \mathrm{~kg} / \mathrm{m}^{2}$, had at least one patent fallopian tube and a normal uterine cavity, whose male partner had a total progressive motile sperm count after washing of the sample after preparation of seminal fluid for IUI insemination of at least $1 \mathrm{million} / \mathrm{mL}$, received ovulation induction through subcutaneous FSH injections with either purified FSH (Foliculin Urofollitropin; Bharat Serums and Vaccines, Maharashtra, India) or recombinant FSH (Gonal-f, Follitropin Alfa; Merck Serono, Feltham, UK), and had basal FSH levels less than $10 \mathrm{IU} / \mathrm{L}$

We randomized the patients into two groups, based on a computer-generated randomization sequence, to receive either IUI with trans- abdominal ultrasound guidance or IUI without ultrasound guidance.

\section{Controlled ovarian stimulation}

All patients in our study started ovulation stimulation on day 2 or day 3 of the menstrual cycle. The initial dose of FSH injections (50$150 \mathrm{IU})$ was determined based on the patient's age, antral follicle count, and any previous IUI cycle response if available. The first transvaginal scan was done on day 2 or 3 of the menstrual cycle and repeated 6 to 8 days later to monitor the ovarian response and endometrial lining. Depending on the ovarian response to stimulation, the dosage of medication was adjusted and thereafter the transvaginal scans were repeated at 2- to 4-day intervals. When the leading follicle reached a diameter of $\geq 17 \mathrm{~mm}$ and the endometrial lining reached a thickness of $\geq 7 \mathrm{~mm}, 10,000 \mathrm{IU}$ of subcutaneous human chorionic gonadotropin (Pregnyl; Merck Sharp \& Dohme, Kenilworth, $\mathrm{NJ}$, USA) was administered to induce an LH surge and the IUI procedure was done 36 hours later. The treatment cycle was cancelled if there were more than three follicles measuring 17 to $20 \mathrm{~mm}$, as this would lead to an increased risk of multiple pregnancy and ovarian hyperstimulation syndrome $[12,13]$.

All semen samples were prepared using the density gradient method (Sil-Select Plus FertiPro, Beernem, Belgium), as described in the 2010 WHO laboratory manual [14]. In both groups, patients were advised to have a full bladder prior to the insemination procedure, and the same intrauterine catheter (Insemi-Cath J-IUIC-351351 3.5 Fr/13 $\mathrm{cm}$; Cook Medical, Bloomington, IN, USA) was used in both groups.

\section{Providers}

To standardize the providers for the IUI procedure and transabdominal pelvic ultrasound, we included one senior clinician and two senior nurses in our study to perform the procedures.

\section{The US-IUI group}

In the US-IUI group, the intrauterine catheter was maneuvered through the cervical canal while the assistant visualized the cervix and the uterus using a transabdominal ultrasound scan (Toshiba Model UDSR-550A Nemio 30, Tokyo, Japan). The clinician performing the insemination maneuvered the catheter to locate the internal cervical opening and directed the catheter into the uterine cavity with the assistance of the ultrasound image. Once the catheter tip was seen passing through the internal cervical opening and the position of the catheter tip was confirmed to be in the uterine cavity, the clinician then flushed the syringe containing the prepared semen into the uterine cavity.

\section{The BM-IUI group}

In the BM-IUI group, the catheter was introduced into the cervical 
canal and maneuvered gently till the resistance of the internal cervical opening was felt to enter the uterine cavity. This was a blind method that depended on the clinician's tactile senses to judge when the transfer catheter was in the correct position. The clinician felt a "give" when the catheter passed through the internal cervical opening, and then flushed the seminal fluid into the uterine cavity.

\section{Luteal support}

For both groups, vaginal luteal phase support with micronized progesterone (200 mg, at 12-hour intervals) (Uterogestan; Besins Healthcare, Bruxelles, Belgium) was started on the day of IUI and continued until a urine pregnancy test was done 3 weeks after the IUI procedure. If the patient had a positive urine pregnancy test, vaginal luteal phase support was replaced with oral dydrogesterone (Duphaston, 10 mg, twice a day; Abbott, Chicago, IL, USA) till 12 weeks of gestation.

\section{IUI cycle outcomes}

A positive urine pregnancy test done 3 weeks after the IUI procedure was considered to indicate pregnancy. Clinical pregnancy was confirmed via an ultrasound scan 2 weeks later with the presence of crown rump length and fetal heart activity.

The degree to which patients felt the IUI procedure to be tolerable was assessed based on a pain visual analog scale (VAS) ranging from 1 (least pain) to 10 (severe pain). The patients were assessed using the pain VAS at the end of the insemination procedure in both groups.

To assess whether US-IUI made the procedure easier for the clinician to perform compared to BM-IUI, we assessed the duration of the IUI insemination procedure, presence or absence of blood stains at the catheter tip after the insemination procedure, whether a vulsellum or tenaculum was necessary for assistance during the procedure, and the level of difficulty (easy or difficult) according to the provider who performed the IUI procedure.

The time taken for the IUI procedure was measured using a digital stop-watch from the introduction of the IUI catheter into the vagina till the IUI catheter was maneuvered into the uterine cavity and the prepared semen was flushed into the uterine cavity.

The level of difficulty was categorized as easy or difficult. If the provider was able to cannulate the catheter into the cervical os in the first attempt, the procedure was labeled as easy. If the provider was not successful with the first attempt, assistance was sought from a second provider, and if the procedure was also difficult for the second provider, then the procedure was labeled as difficult. This classification system was used to standardize whether a procedure was considered to have been difficult.

\section{Statistical analysis}

Continuous data were expressed as mean and standard deviation, or as median and interquartile range, while categorical data were described in the form of frequency and percentage. Differences between the US-IUI and BM-IUI groups in the patients' demographics, IUI treatment procedure, clinical outcomes, clinical pregnancy rate, patient tolerability, and the assessment of the difficulty of the IUI insemination procedure were assessed using the independent $t$-test or the Mann-Whitney U-test for continuous variables and the Pearson chi-square or Fisher exact test for categorical variables. Odds ratios for clinical pregnancy according to type of IUI treatment were calculated using multiple logistic regression, adjusting for the patient's age, body mass index, basal FSH level, indication for IUI (anovulation or tubal factor infertility), the number of days of stimulation, the total drugs for stimulation, endometrial lining on the trigger day, postwash sperm concentration, post-wash motility, post-wash total motile sperm count, and the IUI cycle number. All $p$-values reported were two-sided, and $p$-values $<0.05$ were considered to indicate statistical significance. Analyses were performed using IBM SPSS ver. 20.0 (IBM Corp., Armonk, NY, USA).

\section{Results}

A total of 130 patients were included in our study, of whom 70 underwent ultrasound-guided IUI and 60 patients underwent IUI without ultrasound guidance. The patients' demographics and details of the IUI treatment cycle are summarized in Table 1.

The average age (32.68 years), body mass index $\left(25.12 \mathrm{~kg} / \mathrm{m}^{2}\right)$, day 2-3 basal FSH level (6.48 IU/L), and basal LH level (4.3 IU/L) were similar in both groups. The most common indication for IUI in both groups was unexplained infertility.

The mean total dosage of ovulation stimulation drugs used was 675 IU in the US-IUI group and 800 IU in the BM-IUI group; although the dosage was higher in the BMI-IUI group, this difference was not significant $(p=0.151)$. There was also no significant difference between the groups in the total number of days of stimulation $(p=0.516)$. During IUI stimulation, $62.3 \%$ of the patients had a single dominant follicle with a diameter greater than $17 \mathrm{~mm}$ and 36.2\% had two dominant follicles with a diameter greater than $17 \mathrm{~mm}$, which was similar in both the groups. The uterine endometrial lining was on average 8.7 $\mathrm{mm}$ in both groups $(p=0.145)$.

The post-wash semen concentration was average 61.5 million $/ \mathrm{mL}$, post-wash progressive motility was $89 \%$, the post-wash total motile sperm count was 26.9 million/mL and the percentage of spermatozoa with high-quality post-wash morphology was $4 \%$. There was no significant difference in the post-wash seminal fluid parameters between both groups. 
Table 1. Distribution of demographic characteristics and details of IUI treatment cycles

\begin{tabular}{|c|c|c|c|c|}
\hline Variable & All $(n=130)$ & US-IUI $(n=70)$ & BM-IUI $(n=60)$ & $p$-value \\
\hline Age (yr) & $32.68 \pm 3.30(25-40)$ & $32.29 \pm 3.23(25-40)$ & $33.15 \pm 3.35(26-40)$ & $0.138^{\mathrm{a})}$ \\
\hline Body mass index $\left(\mathrm{kg} / \mathrm{m}^{2}\right)$ & $25.12 \pm 4.27(14.0-35.0)$ & $25.08 \pm 4.25(14.0-35.0)$ & $25.17 \pm 4.39(17.9-34.7)$ & $0.905^{\mathrm{a})}$ \\
\hline Basal FSH (IU/L) & $6.48 \pm 1.52(1.80-9.90)$ & $6.51 \pm 1.45(1.80-9.90)$ & $6.44 \pm 1.62(2.50-9.90)$ & $0.810^{\mathrm{a})}$ \\
\hline Basal LH (IU/L) & & & & $0.983^{b)}$ \\
\hline Median (IQR) & $4.3(3.3-6.0)$ & $4.2(3.3-6.0)$ & $4.3(3.2-6.1)$ & \\
\hline Range & $1.4-16.7$ & $1.4-14.7$ & $1.5-16.7$ & \\
\hline IUl indication & & & & $0.578^{c)}$ \\
\hline Unexplained & $54(41.5)$ & $27(38.6)$ & $27(45.0)$ & \\
\hline Anovulation & $38(29.2)$ & $21(30.0)$ & $17(28.3)$ & \\
\hline Tubal factor & $15(11.5)$ & $11(15.7)$ & $4(6.7)$ & \\
\hline Mild male factor & $7(5.4)$ & $2(2.9)$ & $5(8.3)$ & \\
\hline Endometriosis & $4(3.1)$ & $2(2.9)$ & $2(3.3)$ & \\
\hline Tubal and mild male factor & $3(2.3)$ & $2(2.9)$ & $1(1.7)$ & \\
\hline Anovulation and mild male factor & $4(3.1)$ & $3(4.3)$ & $1(1.7)$ & \\
\hline Anovulation and tubal factor & $5(3.8)$ & $2(2.9)$ & $3(5.0)$ & \\
\hline Dosage of FSH (IU) & & & & $0.057^{b)}$ \\
\hline Median (IQR) & $75.0(75.0-75.0)$ & $75.0(75.0-75.0)$ & $75.0(75.0-75.0)$ & \\
\hline Range & $50.0-150.0$ & $50.0-150.0$ & $50.0-150.0$ & \\
\hline No. of days of stimulation & & & & $0.516^{\mathrm{b})}$ \\
\hline Median (IQR) & $9.5(8.0-12.0)$ & $9.0(8.0-12.0)$ & $10.0(8.0-12.0)$ & \\
\hline Range & $1.0-20.0$ & $1.0-20.0$ & 7.0-19.0 & \\
\hline Total drug dosage for stimulation (IU) & & & & $0.151^{b)}$ \\
\hline Median (IQR) & $750.0(600.0-900.0)$ & $675.0(600.0-900.0)$ & $800.0(600.0-956.2)$ & \\
\hline Range & $400.0-2,250.0$ & $400.0-2,250.0$ & $500.0-1,900.0$ & \\
\hline Endometrial lining on trigger day & & & & $0.145^{b)}$ \\
\hline Median (IQR) & $8.7(7.3-10.3)$ & $8.4(7.2-10.0)$ & $8.9(7.4-11.0)$ & \\
\hline Range & $5.5-15.4$ & $5.5-13.4$ & $5.6-15.4$ & \\
\hline No. of follicles $\geq 17 \mathrm{~mm}$ & & & & $0.792^{\mathrm{c})}$ \\
\hline 1 & $81(62.3)$ & $42(60.0)$ & $39(65.0)$ & \\
\hline 2 & $47(36.2)$ & $27(38.6)$ & $20(33.3)$ & \\
\hline 3 & $2(1.5)$ & $1(1.4)$ & $1(1.7)$ & \\
\hline \multicolumn{5}{|l|}{ Post-wash seminal fluid parameter } \\
\hline Sperm concentration (millions $/ \mathrm{mL}$ ) & & & & $0.152^{\mathrm{b})}$ \\
\hline Median (IQR) & $61.5(25.5-98.0)$ & $66.0(26.7-114.5)$ & $58.5(17.7-85.5)$ & \\
\hline Range & $1.4-429.0$ & $1.8-429.0$ & $1.4-215.0$ & \\
\hline Motility (A+B) (\%) & & & & $0.752^{\mathrm{b})}$ \\
\hline Median (IQR) & $89.0(76.0-93.2)$ & $89.0(76.7-94.0)$ & $89.0(75.2-93.0)$ & \\
\hline Range & $19.0-100.0$ & $19.0-100.0$ & $25.0-100.0$ & \\
\hline Total motile sperm count (millions $/ \mathrm{mL}$ ) & & & & $0.168^{\mathrm{b})}$ \\
\hline Median (IQR) & $26.9(8.5-43.9)$ & $27.5(11.8-48.1)$ & $24.0(6.4-38.5)$ & \\
\hline Range & $1.2-107.0$ & $1.2-107.0$ & $1.2-92.5$ & \\
\hline Morphology (\%) & & & & $0.380^{\mathrm{b})}$ \\
\hline Median (IQR) & $4.0(2.0-5.0)$ & $4.0(2.0-5.0)$ & $3.0(2.0-5.0)$ & \\
\hline Range & $1.0-15.0$ & $1.0-15.0$ & $1.0-12.0$ & \\
\hline
\end{tabular}

Values are presented as mean \pm standard deviation (range) or number (\%) unless otherwise indicated.

$\mathrm{IUI}$, intrauterine insemination; US, ultrasound; BM, blind method; $\mathrm{FSH}$, follicle-stimulating hormone; LH, luteinizing hormone; IQR, interquartile range (25th75th percentile); $A$, progressive linear motile sperm; $B$, nonlinear motile sperms.

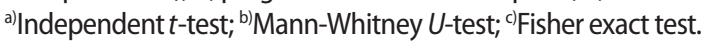

The clinical outcomes are summarized in Table 2. The clinical pregnancy rate was $10 \%$ in both groups $(p>0.995)$. When we compared the patient's pain VAS scores for the insemination procedure, the me- dian pain score was higher in the BM-IUI group than in the US-IUI group (3 vs. 2 , respectively); however, the difference was not significant $(p=0.175)$. 
Table 2. Clinical outcomes

\begin{tabular}{|c|c|c|c|c|}
\hline Variable & All $(n=130)$ & US-IUI $(n=70)$ & BM-IUI $(n=60)$ & $p$-value \\
\hline Clinical pregnancy & $13(10.0)$ & $7(10.0)$ & $6(10.0)$ & $>0.995^{\mathrm{a})}$ \\
\hline Pain VAS score & & & & $0.175^{\mathrm{b})}$ \\
\hline Median (IQR) & $2.0(1.0-4.0)$ & $2.0(1.0-4.0)$ & $3.0(2.0-4.0)$ & \\
\hline Range & $1.0-9.0$ & $1.0-9.0$ & $1.0-7.0$ & \\
\hline Duration (sec) & & & & $0.886^{\mathrm{b})}$ \\
\hline Median (IQR) & $40.0(20.0-60.0)$ & $40.0(24.7-60.0)$ & $43.5(18.5-60.0)$ & \\
\hline Range & $10.0-150.0$ & $10.0-150.0$ & $10.0-120.0$ & \\
\hline Any bleeding & & & & $0.505^{\mathrm{a})}$ \\
\hline Yes & $18(13.8)$ & $11(15.7)$ & 7 (11.7) & \\
\hline No & $112(86.2)$ & $59(84.3)$ & $53(88.3)$ & \\
\hline Difficulty level & & & & $>0.995^{c)}$ \\
\hline Easy & $124(95.4)$ & 67 (95.7) & $57(95.0)$ & \\
\hline Difficult & $6(4.6)$ & $3(4.3)$ & $3(5.0)$ & \\
\hline
\end{tabular}

Values are presented as number (\%) unless otherwise indicated.

US, ultrasound; IUI, intrauterine insemination; BM, blind method; VAS, visual analog scale; IQR, interquartile range (25th-75th percentile).

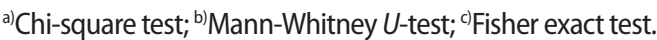

Table 3. Clinical outcomes based on IUI cycle number

\begin{tabular}{|c|c|c|c|}
\hline Variable & US-IUI & BM-IUI & $p$-value \\
\hline Cycle 1 & & & $0.389^{a)}$ \\
\hline Number & 40 & 29 & \\
\hline Clinical pregnancy & $4(10.0)$ & $1(3.4)$ & \\
\hline Pain VAS score & & & $0.911^{b)}$ \\
\hline Median (IQR) & $2.0(1.2-3.0)$ & $2.0(1.0-4.0)$ & \\
\hline Range & $1.0-8.0$ & $1.0-7.0$ & \\
\hline \multicolumn{4}{|l|}{ Level of difficulty } \\
\hline Easy & $38(95.0)$ & $28(96.6)$ & $>0.995^{\mathrm{a})}$ \\
\hline Difficult & $2(5.0)$ & $1(3.4)$ & \\
\hline \multicolumn{4}{|l|}{ Cycle 2} \\
\hline Number & 16 & 20 & \\
\hline Clinical pregnancy & $2(12.5)$ & $3(15.0)$ & $>0.995^{\mathrm{a}}$ \\
\hline Pain VAS score & & & $0.109^{b)}$ \\
\hline Median (IQR) & $2.0(1.0-3.7)$ & $3.0(2.0-4.0)$ & \\
\hline Range & $1.0-5.0$ & $1.0-5.0$ & \\
\hline Level of difficulty & & & $0.492^{\mathrm{a})}$ \\
\hline Easy & $16(100.0)$ & $18(90.0)$ & \\
\hline Difficult & 0 & $2(10.0)$ & \\
\hline \multicolumn{4}{|l|}{ Cycle 3} \\
\hline Number & 13 & 11 & \\
\hline Clinical pregnancy & 0 & $2(18.2)$ & $0.199^{a)}$ \\
\hline Pain VAS score & & & $0.745^{b)}$ \\
\hline Median (IQR) & $2.0(1.0-4.5)$ & $3.0(2.0-4.0)$ & \\
\hline Range & $1.0-9.0$ & $1.0-6.0$ & \\
\hline Level of difficulty & & & $>0.995^{\mathrm{a})}$ \\
\hline Easy & $12(92.3)$ & $11(100.0)$ & \\
\hline Difficult & $1(7.7)$ & 0 & \\
\hline
\end{tabular}

Values are presented as number (\%) unless otherwise indicated.

IUI, intrauterine insemination; US, ultrasound; BM, blind method; VAS, visual analog scale; IQR, interquartile range (25th-75th percentile).

${ }^{\text {a) } F i s h e r ' s ~ e x a c t ~ t e s t ; ~}{ }^{\text {b) }}$ Mann-Whitney $U$-test.
The average duration of the IUI procedure was longer in the BM-IUI group than in the US-IUI group (43.5 seconds vs. 40.0 seconds, respectively), but this difference was not significant $(p=0.886)$. Blood stains were present on the catheter tip in $15.7 \%$ of cases in the US-IUI group and in $11.7 \%$ of cases in the BM-IUI group, which was also not a significant difference $(p=0.505)$. It was not necessary to use a tenaculum or vulsellum during the insemination procedure in any of the cases in either group. The level of difficulty during the procedure was rated as easy in $95.7 \%$ and $95.0 \%$ (and difficult in the remaining $4.3 \%$ and $5 \%$ of cases) in the US-IUI group and in the BM-IUI group, respectively. There was no significant difference in the level of difficulty between the two groups ( $p>0.995$ ).

The clinical outcomes according to the IUI cycle number are summarized in Table 3. In our study, $53.1 \%$ of the patients were in their first IUI cycle, $27.7 \%$ in their second IUI cycle, and $18.5 \%$ in their third IUI cycle. One patient was in her fourth IUI cycle in the US-IUI group.

We conducted a subgroup analysis to compare the clinical pregnancy rate, pain VAS score, and level of difficulty during the IUI procedure for patients undergoing their first IUI cycle or a repeated IUI cycle. The results of the subgroup analysis are summarized in Table 3. Even when comparing patients with the same IUI cycle number, there were still no significant differences between the US-IUI group and the BM-IUI group.

The multivariate analysis showed that there was no significant increase in the clinical pregnancy rate (adjusted odds ratio, 1.07; 95\% confidence interval, $0.85-1.34 ; p=0.558$ ) in the US-IUI group compared to the BM-IUI group (reference) after adjusting for potential covariates such as the patient's age, body mass index, basal FSH level, indication for IUI (anovulation or tubal factor infertility), number of 
Table 4. Multivariate model for clinical pregnancy adjusted for confounding variables

\begin{tabular}{|c|c|c|c|c|}
\hline \multirow{2}{*}{ Variable } & \multicolumn{2}{|c|}{ Clinical pregnancy } & \multirow{2}{*}{ Adjusted OR $(95 \% \mathrm{Cl})^{\mathrm{a})}$} & \multirow{2}{*}{$p$-value } \\
\hline & Yes $(n=13)$ & No $(n=117)$ & & \\
\hline Procedure & & & & 0.558 \\
\hline US-IUI & $7(53.8)$ & $63(53.8)$ & $1.07(0.85-1.34)$ & \\
\hline BM-IUI & $6(46.2)$ & $54(46.2)$ & 1 & \\
\hline
\end{tabular}

Values are presented as number (\%).

$\mathrm{OR}$, odds ratio; $\mathrm{Cl}$, confidence interval; US, ultrasound; IUI, intrauterine insemination; $\mathrm{BM}$, blind method.

a)Adjusted for patient's age, body mass index, basal follicle-stimulating hormone level, indication for IUI (anovulation and tubal factor), number of days of stimulation, total drug dosage for stimulation, endometrial lining on trigger day, post-wash sperm concentration, post-wash sperm motility, post-wash total motile sperm count, and the IUI cycle number.

days of stimulation, total drug dosage for stimulation, endometrial lining on the trigger day, post-wash sperm concentration, post-wash sperm motility, post-wash total motile sperm count, and the IUI cycle number (Table 4).

Thus, there were no significant differences in the clinical pregnancy rate or patient tolerability as assessed by the pain VAS score, and USIUI did not make the insemination procedure easier for clinicians compared to the BM-IUI procedure.

\section{Discussion}

In our study, the clinical pregnancy rate was the same in both groups, and using transabdominal ultrasound for insemination provided no added benefits to the clinicians or patients. For embryo transfer in IVF, ultrasound guidance leads to an increase in the pregnancy and live birth rates [11]. In IVF, the cervical and uterine manipulations during catheterization can induce contractions and endometrial trauma that may lead to expulsion of the embryo [15-17]. Thus, the same principle was applied in our study to reduce the manipulations and trauma caused by the insemination catheter with ultrasound guidance, with the goal of increasing the concentration of sperm in the uterine cavity. In embryo transfer, the embryo is placed 1.0 to $1.5 \mathrm{~cm}$ from the uterine fundus under ultrasound guidance to increase the pregnancy rate $[18,19]$; however, in IUI, the position of the catheter tip in the uterine cavity does not influence the pregnancy rate significantly, as the motile sperm deposited in the uterine cavity need to reach the mature egg in the fallopian tube for fertilization.

Another possible reason why there were no significant betweengroup differences may have been that in both groups, patients were required to have a full bladder prior to the IUI procedure, which is not routinely done for IUI procedures. A full bladder straightens the cervicouterine angle and facilitates insertion of the IUI catheter. This technique has been proven to be effective in embryo transfer, as a full bladder assists in visualization of the uterine lining and insertion of the embryo at the required distance from the uterine fundus [20]. Thus, the presence of a full bladder in both groups could have made it easier for the IUI catheter to be inserted, contributing to the absence of a significant difference in the outcome.

In 2009, Ramon et al. [21] published an RCT with 73 patients to ascertain whether ultrasound guidance during insemination in IUI treatment could increase the pregnancy rate, and concluded that ultrasound did not produce better results than blind insemination, as the pregnancy rates were similar in both groups, which is in accordance with our results. Three years later, Oztekin et al. [22] conducted a retrospective study of $267 \mathrm{IUI}$ cycles in patients with unexplained infertility and concluded that ultrasound-guided IUI increased the pregnancy rates and reduced the frequency of difficult IUI. However, that was a retrospective study and therefore needs to be interpreted with caution. In 2013, Oruc et al. [23] published an RCT with 387 IUI cycles for unexplained infertility, concluding that significantly higher pregnancy rates were achieved only when a senior experienced provider performed ultrasound-guided IUI. In our study, experienced providers performed the ultrasound and the insemination procedure, and we had similar results in both groups. In 2015, Polat et al. [24] carried out a study similar to ours that had 130 patients, and they reported clinical pregnancy rates that were similar to ours.

A drawback of our study is that we recruited patients both in their first IUI cycle and in repeated IUI cycles, meaning that we were unable to determine whether the results of our study are applicable to all patients or whether ultrasound guidance could be useful for patients with a previous difficult IUI. Whether ultrasound guidance increases the pregnancy rate in patients in whom IUI was previously difficult needs further evaluation. We had to discontinue our study after the interim analysis, because there was no difference between the groups, and it took more skilled and experienced personnel to perform the ultrasound inseminations, with an increased cost that did not correspond to an increase in the clinical pregnancy rate.

To our knowledge, our study is the only RCT done so far that has compared patient tolerability and assessed whether the IUI procedure 
was easier for the provider under ultrasound guidance. Our study is also the only RCT that has analyzed patients undergoing their first IUI cycle and repeated IUI cycles separately in a subgroup analysis. Thus, our study clearly showed that that ultrasound-guided insemination did not increase the clinical pregnancy rate in all cases of IUI; however, whether it may increase the clinical pregnancy rate in patients in whom IUI was previously difficult needs further evaluation.

\section{Conflict of interest}

No potential conflict of interest relevant to this article was reported.

\section{Acknowledgments}

The authors would like to thank the Director General of Health Malaysia for the permission to publish this paper.

\section{References}

1. Elguero S, Forman M. Treatment options: II. intrauterine insemination. In: Bayer SR, Alper MM, Penzias AS, editors. The Boston IVF handbook of infertility: a practical guide for practitioners who care for infertile couples. 4th ed. Bra Raton: CRC Press; 2018. p. 72-7.

2. ESHRE Capri Workshop Group. Intrauterine insemination. Hum Reprod Update 2009;15:265-77.

3. Jain S. Intrauterine insemination: current place in infertility management. Eur Med J 2018;3:58-66.

4. Ombelet $\mathrm{W}$. The revival of intrauterine insemination: evidencebased data have changed the picture. Facts Views Vis Obgyn 2017;9:131-2.

5. National Collaborating Centre for Women's and Children's Health. Fertility: assessment and treatment for people with fertility problems. London: the Royal College of Obstetricians and Gynaecologists; 2013.

6. Farquhar CM, Liu E, Armstrong S, Arroll N, Lensen S, Brown J. Intrauterine insemination with ovarian stimulation versus expectant management for unexplained infertility (TUI): a pragmatic, open-label, randomised, controlled, two-centre trial. Lancet 2018;391:441-50.

7. Duran HE, Morshedi M, Kruger T, Oehninger S. Intrauterine insemination: a systematic review on determinants of success. Hum Reprod Update 2002;8:373-84.

8. Lee J, Hwang S, Lee J, Yoo J, Jang D, Hwang K, et al. Effect of insemination timing on pregnancy outcome in association with female age, sperm motility, sperm morphology and sperm concentration in intrauterine insemination. J Obstet Gynaecol Res
2018;44:1100-6.

9. Fancsovits P, Toth L, Murber A, Szendei G, Papp Z, Urbancsek J. Catheter type does not affect the outcome of intrauterine insemination treatment: a prospective randomized study. Fertil Steril 2005;83:699-704.

10. Lemmens $L$, Kos S, Beijer C, Braat DD, Nelen WL, Wetzels AM, et al. Techniques used for IUI: is it time for a change? Hum Reprod 2017;32:1835-45.

11. Brown J, Buckingham K, Buckett W, Abou-Setta AM. Ultrasound versus 'clinical touch' for catheter guidance during embryo transfer in women. Cochrane Database Syst Rev 2016;3:CD006107.

12. Dickey RP, Taylor SN, Lu PY, Sartor BM, Rye PH, Pyrzak R. Risk factors for high-order multiple pregnancy and multiple birth after controlled ovarian hyperstimulation: results of 4,062 intrauterine insemination cycles. Fertil Steril 2005;83:671-83.

13. Berkovitz A, Biron-Shental T, Pasternak Y, Sharony R, Hershko-KIement $A$, Wiser $A$. Predictors of twin pregnancy after ovarian stimulation and intrauterine insemination in women with unexplained infertility. Hum Fertil (Camb) 2017;20:200-3.

14. World Health Organization. WHO laboratory manual for the Examination and processing of human semen. 5th ed. Geneva: World Health Organization; 2010.

15. Practice Committee of the American Society for Reproductive Medicine. Performing the embryo transfer: a guideline. Fertil Steril 2017;107: 882-96.

16. Prapas Y, Prapas N, Hatziparasidou A, Vanderzwalmen P, Nijs M, Prapa $S$, et al. Ultrasound-guided embryo transfer maximizes the IVF results on day 3 and day 4 embryo transfer but has no impact on day 5. Hum Reprod 2001;16:1904-8.

17. Coroleu B, Barri PN, Carreras O, Martinez F, Veiga A, Balasch J. The usefulness of ultrasound guidance in frozen-thawed embryo transfer: a prospective randomized clinical trial. Hum Reprod 2002;17:2885-90.

18. Pope CS, Cook EK, Arny M, Novak A, Grow DR. Influence of embryo transfer depth on in vitro fertilization and embryo transfer outcomes. Fertil Steril 2004;81:51-8.

19. Rovei V, Dalmasso P, Gennarelli G, Lantieri T, Basso G, Benedetto $C$, et al. IVF outcome is optimized when embryos are replaced between 5 and $15 \mathrm{~mm}$ from the fundal endometrial surface: a prospective analysis on 1184 IVF cycles. Reprod Biol Endocrinol 2013;11:114.

20. Mains L, Van Voorhis BJ. Optimizing the technique of embryo transfer. Fertil Steril 2010;94:785-90.

21. Ramon O, Matorras R, Corcostegui B, Meabe A, Burgos J, Exposito $A$, et al. Ultrasound-guided artificial insemination: a randomized controlled trial. Hum Reprod 2009;24:1080-4.

22. Oztekin D, Ozcinar E, Kose C, Gulhan I, Ozeren M, Tinar S. The use 
of ultrasound during intrauterine insemination in unexplained infertility may improve pregnancy outcomes. Med Princ Pract 2013;22:291-4.

23. Oruc AS, Yilmaz N, Gorkem U, Inal HA, Seckin B, Gulerman C. Influence of ultrasound-guided artificial insemination on pregnancy rates: a randomized study. Arch Gynecol Obstet 2014;289:
207-12.

24. Polat I, Ekiz A, Yildirim G, Sahin O, Ulker V, Alkis I, et al. Ultrasound-guided intrauterine insemination versus blind intrauterine insemination: a randomized controlled trial. Clin Exp Obstet Gynecol 2015;42:657-62. 